\title{
Pell-Lucas Series Solution for Fredholm Integral Equations of the Second Kind
}

\author{
Lukonde Peter Alpha \\ Mulungushi University, School of Science Engineering and Technology, Department of Sciences and Mathematics, P.O. Box 80415, \\ Kabwe, Zambia \\ Corresponding author: peterlukonde6.pal[at]gmail.com
}

\begin{abstract}
In this study, a Pell-Lucas series method is introduced to solve Fredholm integral equations of the second kind. The numerical examples show that the proposed method provides highly accurate solutions. Besides, the accuracy of the solutions is checked via the residual error analysis. Finally, the numerical results obtained by using the Pell-Lucas series method are compared in the tables and figures.
\end{abstract}

Keywords: Pell-Lucas Polynomials and Series, Residual error analysis and Absolute error

\section{Introduction}

The differential (DEs), integral (IEs) and integro-differential equations (IDEs) occur frequently in science and engineering [1]. In general an equation, in which the unknown function is under a sign of integration, is called an integral equation [2]. Integral equations are either Fredholm or Volterra type. In this paper, the main focus is on Fredholm integral equations of the second kind. Integral equations belong to a class of functional integro-differential equations.

Most problems arising in, engineering, natural and social sciences are modelled by either linear or nonlinear integral equations. Integral equations arise naturally in physics, chemistry, biology and engineering modelled by initial value problems for a finite interval [a,b] [3]. Scientists, engineers and mathematicians have taken a keen interest in researching about integral equations, because they have a wide range of applications. Some integral equations are complex to solve using analytical methods, that is why researchers have developed numerical methods to solve integral equations that cannot be solved analytically. In literature, there are many methods for solving Fredholm integral equations. In particular Bulent and Yasin [4] presented numerical methods for solving Fredholm integral equations of the second type. They compared the results of four different techniques, which are Bernstein Piecewise Polynomials Method (BPPM), Integral Mean Value Method (IMVM), Taylor Series Method (TSM) and the Least Square Method (LSM). These methods appeared to be very useful and effective for finding approximate solutions of Fredholm integral equations of the second type. Firouzdor et al [5] proposed a numerical method for solving integral equations, this method is used for both Fredholm and Volterra integral equations. Huaiqing et al [6] presented a method for solving linear integral equations using radial basis function interpolation. Other methods include, numerical approximation of Fredholm integral equation (FIE) of the second kind using Galerkin and collocation method, numerical solution for Fredholm-Volterra integral equation of the second kind using collocation and Galerkin methods, iterated Petrov-Galerkin with regular pairs for solving Fredholm integral equations of the second kind, and collocation technique for numerical solution of integral equations with certain basis function in interval [0,1] [7-11]. Sezer et al [12-16] worked on a number of highly effective methods for solving functional IDEs and their subclasses, such as linear and nonlinear forms.

The aim of this paper is to present a numerical method for solving the Fredholm integral equation of the second kind, using Pell-Lucas series method. The Fredholm integral equation is transformed into the fundamental matrix equation, which is then solved and the required values of the Pell-Lucas series coefficient matrix are found. This method has an advantage over other methods, because it turns the problem into a linear system of equations. Larger matrices are easily solved using computer soft wares such as MATLAB, MAPLE SOFT, and MATEMATICA.

We consider the Fredholm type integral equations are given by

$$
y(x)=f(x)+\lambda \int_{a}^{b} K(x, t) y(t) d t
$$

where $f(x)$ and $K(x, t)$ are continuous functions on the interval $a \leq x, t \leq b$ and $\lambda$ is a known constant. The solution of the Fredholm integral equation given by (1) is found numerically by

$$
y(x) \cong y_{N}(x)=\sum_{n}^{N} a_{n} Q_{n}(x)
$$

where $Q_{n}(x)$ is the Pell-Lucas polynomial and $a_{n}$ is the coefficient of the Pell-Lucas polynomial in the series defined by (2).Pell-Lucas polynomials are given by the recurrence formula $Q_{n}(x)=2 x Q_{n-1}(x)+Q_{n-2}(x)$ or by $Q_{n}(x)=\sum_{k=0}^{\left\lfloor\frac{n}{2}\right\rfloor} 2^{n-2 k} \frac{n}{n-k}\left(\begin{array}{c}n-k \\ k\end{array}\right) x^{n-2 k}$ their derivatives are written as $Q_{n}^{(k)}(x)=\frac{d^{k}}{d x^{k}}\left(2 x Q_{n-1}(x)+Q_{n-2}(x)\right)$ When $x=1, Q_{n}(1)=Q_{n}$, where $Q_{n}$ is the $n$-th Pell-

\section{Volume 10 Issue 6, June 2021 www.ijsr.net}


Lucas number. The first two Pell-Lucas polynomials are $Q_{0}(x)=2$ and $Q_{1}(x)=2 x$.

\section{Important Matrix Relations}

From the approximate solution $y(x)$ of (1) which is given by a truncated Pell-Lucas series (2) for $n=0,1,2, \ldots, N$, we convert the finite series (2) to the matrix form as

$$
y(x) \cong y_{N}(x)=\left[\begin{array}{lllll}
Q_{0}(x) & Q_{1}(x) & Q_{2}(x) & \cdots & Q_{N}
\end{array}\right] \cdot \boldsymbol{A}=\boldsymbol{Q}(x) \boldsymbol{A}
$$

Where, $\quad \boldsymbol{A}=\left[\begin{array}{lllll}a_{0} & a_{1} & a_{2} & \cdots & a_{N}\end{array}\right]^{T}$. Using the definition of the Pell-Lucas polynomials, one obtains the matrix $\boldsymbol{Q}(x)$ as

$$
\boldsymbol{Q}(x)=\boldsymbol{X}(x) \boldsymbol{L}
$$

The matrix relation (4) is then substituted into the matrix relation (3) to get

$$
y(x) \cong y_{N}(x)=\boldsymbol{X}(x) \boldsymbol{L A}
$$

The $\boldsymbol{L}$ in (5) is obtained from $\boldsymbol{L}^{T}$ which is defined as

$$
\boldsymbol{L}^{T}=\left[\begin{array}{cccccc}
2 & 0 & 0 & 0 & \cdots & 0 \\
2^{0} \frac{2}{1}\left(\begin{array}{c}
1 \\
1
\end{array}\right) & 2^{1} \frac{1}{1}\left(\begin{array}{l}
1 \\
0
\end{array}\right) & 0 & 0 & \cdots & 0 \\
0 & 2^{1} \frac{3}{2}\left(\begin{array}{l}
2 \\
1
\end{array}\right) & 0 & 2^{3} \frac{3}{3}\left(\begin{array}{l}
2 \\
2 \\
0
\end{array}\right) & \cdots & 0 \\
\vdots & \vdots & \vdots & \ddots & \vdots \\
0 & 2^{1} \frac{N}{2}\left(\frac{N+1}{2}\left(\frac{N-1}{2}\right)\right. & 0 & 2^{1} \frac{N+3}{2}\left(\frac{N+3}{2}\right) & \cdots & 2^{N} \frac{N}{N}\left(\begin{array}{c}
N \\
0
\end{array}\right)
\end{array}\right]
$$

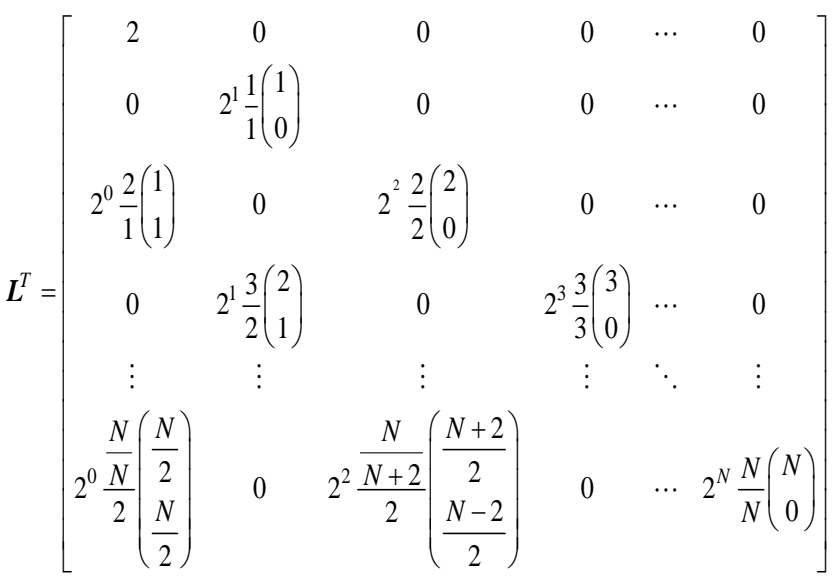

if $N$ is even. We introduce matrix relation of a kernel function as

$$
K(x, t)=\boldsymbol{X}(x) \boldsymbol{K} \boldsymbol{X}^{T}(t)
$$

where,

$$
\boldsymbol{K}=\left[k_{p q}\right] ; k_{p q}=\frac{1}{p ! q !} \frac{\partial^{p+q} K(0,0)}{\partial x^{p} \partial t^{q}} ; p, q=0,1,2, \ldots, N
$$

Using (5) the matrix relation for $y(t)$ is obtained as

$$
y(t)=\boldsymbol{X}(t) \boldsymbol{L A}
$$

Substituting the matrix relations (5), (6) and (7) into Eq. (1) yields

$$
\begin{gathered}
\boldsymbol{X}(x) \boldsymbol{L A}=\boldsymbol{f}(x)+\lambda \int_{a}^{b} \boldsymbol{X}(x) \boldsymbol{K} \boldsymbol{X}^{T}(t) \boldsymbol{X}(t) d t \boldsymbol{L A} \\
\boldsymbol{X}(x) \boldsymbol{L A}=\boldsymbol{f}(x)+\lambda \boldsymbol{X}(x) \boldsymbol{K} \underbrace{\int_{a}^{b} \boldsymbol{X}^{T}(t) \boldsymbol{X}(t) d t \boldsymbol{L A}}_{M} \\
\boldsymbol{X}(x) \boldsymbol{L A}=\boldsymbol{f}(x)+\lambda \boldsymbol{X}(x) \boldsymbol{K M L A}
\end{gathered}
$$

if $N$ is odd, and

The matrix $\boldsymbol{M}$ is obtained from the calculationof the integral below,

$$
\begin{aligned}
& \boldsymbol{M}=\int_{a}^{b} \boldsymbol{X}^{T}(t) \boldsymbol{X}(t) d t=\int_{a}^{b}\left[\begin{array}{c}
1 \\
\vdots \\
t^{N}
\end{array}\right]\left[\begin{array}{llll}
1 & t & \cdots & t^{N}
\end{array}\right] d t=\int_{a}^{b}\left[t^{i+j}\right] d t ; i, j=0,1, \ldots, N \\
& \boldsymbol{M}=\left[\frac{t^{i+j+1}}{i+j+1}\right]_{a}^{b}=\left[\frac{b^{i+j+1}-a^{i+j+1}}{i+j+1}\right] ; i, j=0,1, \ldots, N \\
& \boldsymbol{X}(x) \boldsymbol{L A}=\boldsymbol{X}(x) \boldsymbol{F}+\lambda \boldsymbol{X}(x) \boldsymbol{K M} \boldsymbol{L A}
\end{aligned}
$$

We now define $f(x)$ using the Maclaurin series as,

$f(x)=\boldsymbol{X}(x) \boldsymbol{F} ; f_{n}=\left[\begin{array}{c}f_{0} \\ \vdots \\ f_{N}\end{array}\right], f_{n}=\frac{f^{(n)}(0)}{n !} ; n=0,1, \ldots, N$

Relation (10) is purely an algebraic equation which can be easily solved. The matrix relation (10) is simplified into the fundamental matrix as, 


$$
\begin{gathered}
\boldsymbol{X}(x) \boldsymbol{L A}-\lambda \boldsymbol{X}(x) \boldsymbol{K M L A}=\boldsymbol{X}(x) \boldsymbol{F} \Rightarrow \boldsymbol{X}(x)\left\{\boldsymbol{I}_{n}-\lambda \boldsymbol{K} \boldsymbol{M}\right\} \boldsymbol{L A}=\boldsymbol{X}(x) \boldsymbol{F} \\
\Rightarrow \boldsymbol{X}^{-1}(x) \boldsymbol{X}(x)\left\{\boldsymbol{I}_{n}-\lambda \boldsymbol{K} \boldsymbol{M}\right\} \boldsymbol{L A}=\boldsymbol{X}^{-1}(x) \boldsymbol{X}(x) \boldsymbol{F} \Rightarrow \\
\underbrace{\left\{\boldsymbol{I}_{n}-\lambda \boldsymbol{K} \boldsymbol{M}\right\} \boldsymbol{L} \boldsymbol{A}=\boldsymbol{F}}_{W}
\end{gathered}
$$

The fundamental matrix (11) can be written in compact form as,

$$
\boldsymbol{W A}=\boldsymbol{F}
$$

or as an augmented matrix shown below,

$$
[\boldsymbol{W} ; \boldsymbol{F}]=\left[\begin{array}{cccccc}
w_{00} & w_{01} & \cdots & w_{0 N} & ; & f_{0} \\
w_{10} & w_{11} & \cdots & w_{1 N} & ; & f_{1} \\
\vdots & \vdots & \ddots & \vdots & ; & \vdots \\
w_{N 0} & w_{N 1} & \cdots & w_{N N} & ; & f_{N}
\end{array}\right]
$$

The matrix relation (12) has a unique solution if and only if its determinant is not equal to zero, thus,

$$
|\boldsymbol{W}| \neq \boldsymbol{O} \Rightarrow \boldsymbol{A}=\boldsymbol{W}^{-1} \boldsymbol{F}
$$

$\boldsymbol{A}$ is the desired coefficient matrix of Pell-Lucas polynomial, therefore the approximate solution (2) now obtained.

\section{Residual Error and Absolute Error Analysis}

In this section, the residual error and the classical absolute error methods are given. When we substitute solution $y_{N}(x)$ into the Eq. (1), the resulting equation is approximately obtained as follows;

$$
R_{N}\left(x_{i}\right)=y_{N}\left(x_{i}\right)-\lambda \int_{a}^{b} K\left(x_{i}, t\right) y_{N}(t) d t-f\left(x_{i}\right) \cong 0
$$

where $x_{i} \in[a, b] ; i=0,1,2, \ldots \quad$ or $\quad R_{N}\left(x_{i}\right) \leq 10^{-k_{i}}$ such that $k_{i}$ is any positive number. Taking the max to be $10^{-k_{i}}=10^{-k}$, then the truncation limit $N$ is increased until the difference $R_{N}\left(x_{i}\right)$ at each point is smaller than the predetermined $10^{-k}$. If $R_{N}\left(x_{i}\right) \rightarrow 0$ and $N$ is sufficiently large, then the error decreases. The accuracy of the solution can be checked and the error can be estimated by the residual function $R_{N}(x)$ as expressed in [1]. Using the inequality $\left|\int_{a}^{b} R_{N}(x) d x\right| \leq \int_{a}^{b}\left|R_{N}(x)\right| d x$ and the integral mean value theorem, the upper bound of the mean error $\bar{R}_{N}$ is

$$
\int^{b}\left|R_{N}(x)\right| d x
$$

obtained as $R_{N}(c) \leq \frac{a}{b-a}=\bar{R}_{N}$. Besides, the absolute error is given by

$R=\mid$ Exact solution - Approximate solution $|=| y(x)-y_{N}(x) \mid$

\section{Numerical Examples}

Example 1: Consider the following Fredholm integral equation,

$$
y(x)=x+\int_{0}^{1}\left(x t-x^{2}\right) y(t) d t, 0 \leq x, t \leq 1 .
$$

The exact solution of Eq. (16) is $y(x)=\frac{96}{73} x-\frac{36}{73} x^{2}$. From Eq. (16), one obtains, $f(x)=x \quad$ and $K(x, t)=x t-x^{2}$ which are continuous functions in the interval $[0,1]$. To find the numerical solution for this problem, the Pell-Lucas series method is used. For $N=2$, the approximate solution is given by,

$$
y(x) \cong y_{2}(x)=\sum_{n=0}^{2} a_{n} Q_{n}(x) .
$$

The fundamental matrix equation for this problem is written as,

$$
\begin{gathered}
\{\boldsymbol{I}-\lambda \boldsymbol{K} \boldsymbol{M}\} \boldsymbol{L A}=\boldsymbol{F}, \text { if } \boldsymbol{W}=\{\boldsymbol{I}-\lambda \boldsymbol{K} \boldsymbol{M}\} \boldsymbol{L} \text { then } \\
\boldsymbol{W A}=\boldsymbol{F}
\end{gathered}
$$

where,

$$
\begin{gathered}
\boldsymbol{K}=\left[\begin{array}{ccc}
0 & 0 & 0 \\
0 & 1 & 0 \\
-1 & 0 & 0
\end{array}\right], \boldsymbol{M}=\left[\begin{array}{ccc}
1 & \frac{1}{2} & \frac{1}{3} \\
\frac{1}{2} & \frac{1}{3} & \frac{1}{4} \\
\frac{1}{3} & \frac{1}{4} & \frac{1}{5}
\end{array}\right], \boldsymbol{I}=\left[\begin{array}{lll}
1 & 0 & 0 \\
0 & 1 & 0 \\
0 & 0 & 1
\end{array}\right], \\
\boldsymbol{L}=\left[\begin{array}{lll}
2 & 0 & 2 \\
0 & 2 & 0 \\
0 & 0 & 4
\end{array}\right], \boldsymbol{F}=\left[\begin{array}{l}
0 \\
1 \\
0
\end{array}\right], \boldsymbol{A}=\left[\begin{array}{c}
a_{0} \\
a_{1} \\
a_{2}
\end{array}\right], \lambda=1
\end{gathered}
$$

After performing the necessary operations on the fundamental matrix equation, the following augmented matrix is obtained;

$$
[\boldsymbol{W} ; \boldsymbol{F}]=\left[\begin{array}{ccccc}
2 & 0 & 2 & ; & 0 \\
-1 & 4 / 3 & -2 & ; & 1 \\
2 & 1 & 22 / 3 & ; & 0
\end{array}\right]
$$

Solving the system of three unknowns in the above matrix yields the matrix $\boldsymbol{A}$ as;

$$
\boldsymbol{A}=\left[\begin{array}{lll}
\frac{9}{73} & \frac{48}{73} & -\frac{9}{73}
\end{array}\right]^{T}
$$

Consequently the numerical solution for Eq. (16) is obtained as; 


\section{International Journal of Science and Research (IJSR)}

$y_{2}(x)=\frac{9}{73} \cdot(2)+\frac{48}{73} \cdot(2 x)+(-) \frac{9}{73} \cdot\left(4 x^{2}+2\right)=\frac{96}{73} x-\frac{36}{73} x^{2}$, this is the same as exact solution.

Now, let us compare the results of the same problem presented by [4] for the same problem. The approximate solutions of the integral equation are obtained using Integral Mean Value Method (IMVM), Bernstein Piecewise Polynomials Method (BPPM), The Least Square Method (LSM), and Taylor Series Method (TSM) respectively.

$\varphi_{I M V M}(x)=1.397922046675241 x-0.6308106266346826 x^{2}$,

$\varphi_{B P P M}(x)=1.3150684931506849 x-0.4931506849315068 x^{2}$,

$\varphi_{L S M}(x)=0.6575342465753424 x+0.4931506849315068 x^{2}$,

$\varphi_{T S M}(x)=1.397922046675241 x-0.6308106266346826 x^{2}$,

$y_{2}(x)=\frac{96}{73} x-\frac{36}{73} x^{2}$, current method.

$$
\begin{aligned}
& \begin{aligned}
N=2 ; y_{2}(x) & =1+\frac{15}{16} x+\frac{1}{2} x^{2} \\
N=3 ; y_{3}(x) & =1+\frac{79}{80} x+\frac{1}{2} x^{2}+\frac{1}{6} x^{3} \\
N=7 ; y_{7}(x)= & 1+0.9999958664 x+0.5000000001 x^{2} \\
& +0.008178323412 x^{5}+0.0013888888
\end{aligned} \\
& N=11 ; y_{11}(x)=0.9999999998+0.9999999998 x+0 \\
& +
\end{aligned}
$$$$
N=7 ; y_{7}(x)=1+0.9999958664 x+0.5000000001 x^{2}+0.1666666666 x^{3}+0.04166666666 x^{4}
$$$$
+0.008178323412 x^{5}+0.001388888889 x^{6}+0.0001984126984 x^{7}
$$$$
N=11 ; y_{11}(x)=0.9999999998+0.99999999998 x+0.4999999816 x^{2}+0.1666666651 x^{3}+0.04166666667 x^{4}
$$$$
+0.008333427792 x^{5}+0.001388888889 x^{6}+0.0001984126984 x^{7}+0.00002480158730 x^{8}
$$

\begin{tabular}{|c|c|c|c|c|c|}
\hline$x_{i}$ & $\begin{array}{l}\text { Exact Solution } \\
y\left(x_{i}\right)=e^{x_{i}}\end{array}$ & $N=2, y_{2}\left(x_{i}\right)$ & $N=3, y_{3}\left(x_{i}\right)$ & $N=7, y_{7}\left(x_{i}\right)$ & $N=11, y_{11}\left(x_{i}\right)$ \\
\hline 0 & 1.000000000 & 1.000000000 & 1.000000000 & 1.000000000 & 0.9999999998 \\
\hline 0.1 & 1.105170918 & 1.098750000 & 1.103916667 & 1.105170504 & 1.105170918 \\
\hline 0.2 & 1.221402758 & 1.207500000 & 1.218833333 & 1.221401882 & 1.221402758 \\
\hline 0.3 & 1.349858808 & 1.326250000 & 1.345750000 & 1.349857189 & 1.349858805 \\
\hline 0.4 & 1.491824698 & 1.455000000 & 1.4856666667 & 1.491821441 & 1.491824696 \\
\hline 0.5 & 1.648721271 & 1.593750000 & 1.639583333 & 1.648714257 & 1.648721268 \\
\hline 0.6 & 1.822118800 & 1.742500000 & 1.808500000 & 1.822103820 & 1.822118801 \\
\hline 0.7 & 2.013752707 & 1.901250000 & 1.993416667 & 2.013722212 & 2.013752713 \\
\hline 0.8 & 2.225540928 & 2.070000000 & 2.195333333 & 2.225482265 & 2.225540948 \\
\hline 0.9 & 2.459603111 & 2.248750000 & 2.415250000 & 2.459496011 & 2.459603151 \\
\hline 1.0 & 2.718281828 & 2.437500000 & 2.654166667 & 2.718094825 & 2.718281903 \\
\hline
\end{tabular}$$
+2.755731922 \cdot 10^{-6} x^{9}+2.755731922 \cdot 10^{-7} x^{10}+2.505210839 \cdot 10^{-8} x^{11} .
$$
calculated absolute errors.

Table 1: Comparison of the exact and numerical solutions of Example 2

Table 2: The comparison of absolute errors corresponding to Example 2

\begin{tabular}{|c|c|c|c|c|}
\hline$x_{i}$ & $\left|e^{x_{i}}-y_{2}\left(x_{i}\right)\right|$ & $\left|e^{x_{i}}-y_{3}\left(x_{i}\right)\right|$ & $\left|e^{x_{i}}-y_{7}\left(x_{i}\right)\right|$ & $\left|e^{x_{i}}-y_{11}\left(x_{i}\right)\right|$ \\
\hline 0 & 0.00000000000000000 & 0.0000000000000000 & 0 & $2 \mathrm{e}-10$ \\
\hline 0.1 & 0.00642091800000011 & 0.00125425099999998 & $4.14 \mathrm{e}-07$ & 0 \\
\hline 0.2 & 0.01390275800000000 & 0.0025694249999999 & $8.76 \mathrm{e}-07$ & 0 \\
\hline 0.3 & 0.02360880800000010 & 0.00410880800000002 & $1.619 \mathrm{e}-06$ & $3 \mathrm{e}-09$ \\
\hline 0.4 & 0.03682469800000000 & 0.00615803100000001 & $3.257 \mathrm{e}-06$ & $2 \mathrm{e}-09$ \\
\hline 0.5 & 0.05497127100000010 & 0.00913793800000007 & $7.014 \mathrm{e}-06$ & $3 \mathrm{e}-09$ \\
\hline 0.6 & 0.07961880000000000 & 0.0136187999999999 & $1.498 \mathrm{e}-05$ & $1 \mathrm{e}-09$ \\
\hline 0.7 & 0.11250270700000000 & 0.0203360400000001 & $3.0495 \mathrm{e}-05$ & $6 \mathrm{e}-09$ \\
\hline 0.8 & 0.15554092800000000 & 0.0302075949999998 & $5.8663 \mathrm{e}-05$ & $2 \mathrm{e}-08$ \\
\hline 0.9 & 0.21085311100000000 & 0.0443531109999999 & $1.071 \mathrm{e}-04$ & $4 \mathrm{e}-08$ \\
\hline 1 & 0.28078182800000000 & 0.0641151609999997 & $1.87 \mathrm{e}-04$ & $7.5 \mathrm{e}-08$ \\
\hline
\end{tabular}

\section{Volume 10 Issue 6, June 2021} www.ijsr.net

Licensed Under Creative Commons Attribution CC BY 


\section{International Journal of Science and Research (IJSR) \\ ISSN: 2319-7064 \\ SJIF (2020): 7.803}

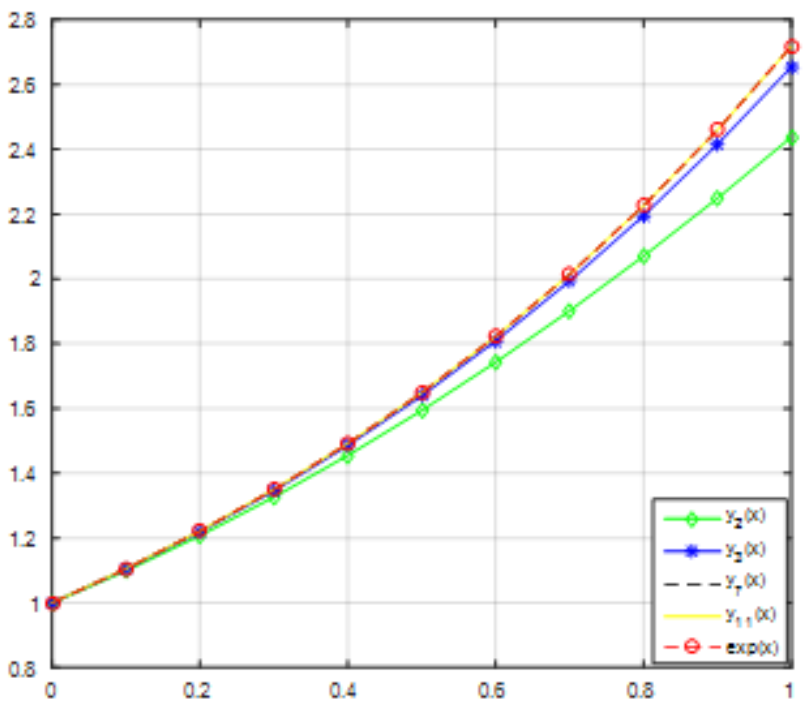

Figure 1: Comparison between exact and numerical solutions of Example 2
Example 3: Consider the following Fredholm integral equation,

$$
y(x)=f(x)+\int_{0}^{1}(1+x t) y(t) d t, 0 \leq x, t \leq 1 .
$$

The exact solution of Eq. (18) is $y(x)=\cos (x)$ From Eq. (18), one obtains, $f(x)=\cos (x)-x \cos (1)-x \sin (1)-\sin (1) \quad$ and $K(x, t)=1+x t$ which are continuous functions in the interval $[0,1]$.The numerical results shown in Table 3, shows the effectiveness of the method as the values of $N$ increases the numerical solution becomes closer and closer to the exact solution. Figure 2and Figure 3 reveals that the approximate solutions are consistent with the exact solution even for the low values of $N$. Figure 4 shows the variation of absolute errors for selected values of $N$.

Table 3: Comparison of the exact and numerical solutions of Example 3

\begin{tabular}{|c|c|c|c|c|c|}
\hline$x_{i}$ & $\begin{array}{c}\text { Exact Solution } \\
\left(x_{i}\right)=\cos \left(x_{i}\right)\end{array}$ & $N=2, y_{2}\left(x_{i}\right)$ & $N=4, y_{4}\left(x_{i}\right)$ & $N=6, y_{6}\left(x_{i}\right)$ & $N=10, y_{10}\left(x_{i}\right)$ \\
\hline 0 & 1.000000000 & 1.0352469860 & 0.9991358739 & 1.000012198 & 1.0000000000 \\
\hline 0.1 & 0.9950041653 & 1.0318745160 & 0.9941009042 & 0.9950169093 & 0.9950041655 \\
\hline 0.2 & 0.9800665778 & 1.0185020470 & 0.9791242678 & 0.9800798678 & 0.9800665786 \\
\hline 0.3 & 0.9553364891 & 0.9951295770 & 0.9543559648 & 0.9553503235 & 0.9553364906 \\
\hline 0.4 & 0.9210609940 & 0.9617571070 & 0.9200459951 & 0.9210753608 & 0.9210609968 \\
\hline 0.5 & 0.8775825619 & 0.9183846380 & 0.8765443587 & 0.8775973943 & 0.8775825661 \\
\hline 0.6 & 0.8253356149 & 0.8650121680 & 0.8243010556 & 0.8253506750 & 0.8253356210 \\
\hline 0.7 & 0.7648421873 & 0.8016396980 & 0.7638660859 & 0.7648567863 & 0.7648421956 \\
\hline 0.8 & 0.6967067093 & 0.7282672280 & 0.6958894495 & 0.6967191448 & 0.6967067201 \\
\hline 0.9 & 0.6216099683 & 0.6448947590 & 0.6211211464 & 0.6216165005 & 0.6216099815 \\
\hline 1.0 & 0.5403023059 & 0.5515222890 & 0.5404111767 & 0.5402954378 & 0.5403023208 \\
\hline
\end{tabular}

Table 4: The comparison of absolute errors corresponding to Example 3

\begin{tabular}{|l|c|l|l|l|}
\hline$x_{i}$ & $\left|\cos \left(x_{i}\right)-y_{2}\left(x_{i}\right)\right|$ & $\left|\cos \left(x_{i}\right)-y_{4}\left(x_{i}\right)\right|$ & $\left|\cos \left(x_{i}\right)-y_{6}\left(x_{i}\right)\right|$ & $\left|\cos \left(x_{i}\right)-y_{10}\left(x_{i}\right)\right|$ \\
\hline 0 & 0.0352469860000000 & 0.000864126099999973 & $1.2198 \mathrm{e}-05$ & 0 \\
\hline 0.1 & 0.0368703507000000 & 0.000903261100000052 & $1.2744 \mathrm{e}-05$ & $2.00 \mathrm{e}-10$ \\
\hline 0.2 & 0.0384354692000000 & 0.000942310000000002 & $1.329 \mathrm{e}-05$ & $8.00 \mathrm{e}-10$ \\
\hline 0.3 & 0.0397930879000000 & 0.000980524300000063 & $1.38344 \mathrm{e}-05$ & $1.50 \mathrm{e}-09$ \\
\hline 0.4 & 0.0406961130000001 & 0.00101499890000001 & $1.43668 \mathrm{e}-05$ & $2.80 \mathrm{e}-09$ \\
\hline 0.5 & 0.0408020761000001 & 0.00103820319999992 & $1.48324 \mathrm{e}-05$ & $4.20 \mathrm{e}-09$ \\
\hline 0.6 & 0.0396765531000001 & 0.00103455929999996 & $1.50601 \mathrm{e}-05$ & $6.10 \mathrm{e}-09$ \\
\hline 0.7 & 0.0367975107000000 & 0.000976101399999929 & $1.4599 \mathrm{e}-05$ & $8.30 \mathrm{e}-09$ \\
\hline 0.8 & 0.0315605187000000 & 0.000817259800000025 & $1.24355 \mathrm{e}-05$ & $1.08 \mathrm{e}-08$ \\
\hline 0.9 & 0.0232847907000000 & 0.000488821899999992 & $6.53 \mathrm{e}-06$ & $1.32 \mathrm{e}-08$ \\
\hline 1 & 0.0112199831000001 & 0.000108870800000105 & $6.8681 \mathrm{e}-06$ & $1.49 \mathrm{e}-08$ \\
\hline
\end{tabular}

\section{Volume 10 Issue 6, June 2021} www.ijsr.net 


\section{International Journal of Science and Research (IJSR) \\ ISSN: 2319-7064 \\ SJIF (2020): 7.803}

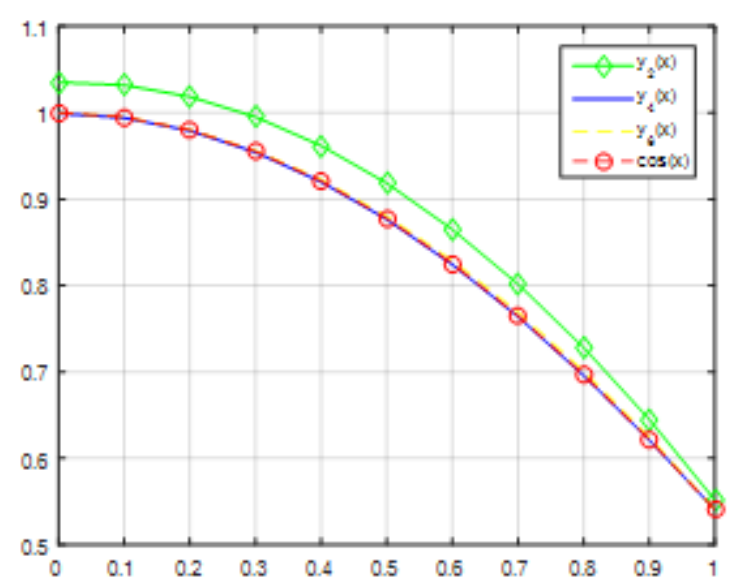

Figure 2: Comparison between exact and numerical solutions of Example 3

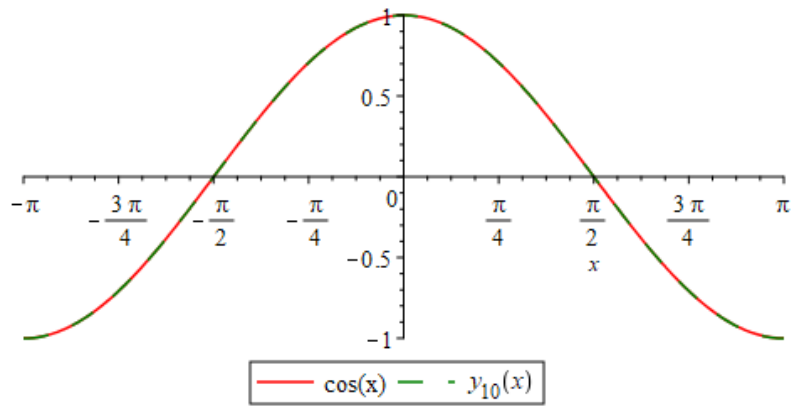

Figure 3: Comparison between exactand the numerical solution of Example 3w hen $N=10$.

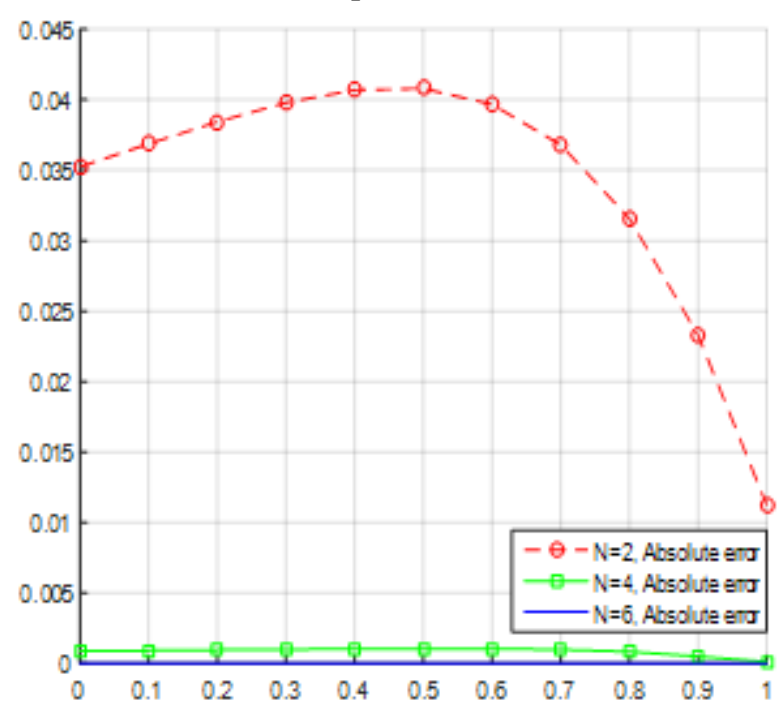

Figure 4: Comparison of absolute errors corresponding to Example 3

Example 4: Let us consider the following Fredholm integral equation,

$$
y(x)=\frac{4 \pi x-\sin \pi x}{2}+\frac{1}{2} \int_{0}^{1} x \cos \left(\pi x t^{2}\right) y(t) d t, 0 \leq x, t \leq 1 .
$$

The exact solution of Eq. (19) is $y(x)=2 \pi x$. From Eq.

(19), one obtains, $f(x)=\frac{4 \pi x-\sin \pi x}{2}$ and $K(x, t)=x \cos \left(\pi x t^{2}\right)$ which are continuous functions in the interval $[0,1]$.The fundamental matrix equation for this problem is written as, $\{\boldsymbol{I}-\lambda \boldsymbol{K} \boldsymbol{M}\} \boldsymbol{L A}=\boldsymbol{F}$, if $\boldsymbol{W}=\{\boldsymbol{I}-\lambda \boldsymbol{K} \boldsymbol{M}\} \boldsymbol{L}$ then $\boldsymbol{W A}=\boldsymbol{F}$

where,

$$
\begin{aligned}
\boldsymbol{K} & =\left[\begin{array}{lll}
0 & 0 & 0 \\
1 & 0 & 0 \\
0 & 0 & 0
\end{array}\right], \boldsymbol{M}=\left[\begin{array}{ccc}
1 & 1 / 2 & 1 / 3 \\
1 / 2 & 1 / 3 & 1 / 4 \\
1 / 3 & 1 / 4 & 1 / 5
\end{array}\right], \boldsymbol{I}=\left[\begin{array}{lll}
1 & 0 & 0 \\
0 & 1 & 0 \\
0 & 0 & 1
\end{array}\right], \\
\boldsymbol{L} & =\left[\begin{array}{lll}
2 & 0 & 2 \\
0 & 2 & 0 \\
0 & 0 & 4
\end{array}\right], \boldsymbol{F}=\left[\begin{array}{c}
0 \\
3 \pi / 2 \\
0
\end{array}\right], \boldsymbol{A}=\left[\begin{array}{c}
a_{0} \\
a_{1} \\
a_{2}
\end{array}\right], \lambda=\frac{1}{2}
\end{aligned}
$$

Performing the necessary operations on the fundamental matrix equation, the augmented matrix is obtained;

$$
[\boldsymbol{W} ; \boldsymbol{F}]=\left[\begin{array}{ccccc}
2 & 0 & 2 & ; & 0 \\
-1 & 3 / 2 & -5 / 2 & ; & 3 \pi / 2 \\
0 & 0 & 4 & ; & 0
\end{array}\right]
$$

Solving the system of three unknowns in the above matrix yields the matrix $\boldsymbol{A}$ as;

$\boldsymbol{A}=\left[\begin{array}{lll}0 & \pi & 0\end{array}\right]^{T}$

Therefore,

$y_{2}(x)=0(2)+\pi \cdot(2 x)+0 \cdot\left(4 x^{2}+2\right)=2 x \pi$, this is the same as exact solution.

The solution of the same problem is presented by [2] as,

$$
\begin{aligned}
y_{0}(x)= & -0.298544 x^{7}+1.27105 x^{5}-2.57756 x^{3}+ \\
& 7.85213 x-0.5 \sin (\pi x)
\end{aligned}
$$

The results of this problem are then compared with the ones presented by [2] in Table 5 and Figure 5.

Table 5: Comparison of the exact and numerical solutions of Example 4

\begin{tabular}{|c|c|c|c|}
\hline$x_{i}$ & $\begin{array}{c}\text { Exact solution } \\
y\left(x_{i}\right)=2 \pi x_{i}\end{array}$ & $\begin{array}{c}\text { Picard-type } \\
\text { iterative scheme } \\
y_{0}\left(x_{i}\right)\end{array}$ & $\begin{array}{c}\text { Current method } \\
N=2, y_{2}\left(x_{i}\right) \\
=2 \pi x_{i}\end{array}$ \\
\hline 0 & 0.0000000000 & 0.0000000000 & 0.0000000000 \\
\hline 0.1 & 0.6283185308 & 0.6281396234 & 0.6283185308 \\
\hline 0.2 & 1.2566370620 & 1.2563158090 & 1.2566370620 \\
\hline 0.3 & 1.8849555920 & 1.8845597430 & 1.8849555920 \\
\hline 0.4 & 2.5132741240 & 2.5128863200 & 2.5132741240 \\
\hline 0.5 & 3.1415926540 & 3.1412579380 & 3.1415926540 \\
\hline 0.6 & 3.7699111840 & 3.7694763090 & 3.7699111840 \\
\hline 0.7 & 4.3982297160 & 4.3969184140 & 4.3982297160 \\
\hline 0.8 & 5.0265482460 & 5.0219891030 & 5.0265482460 \\
\hline 0.9 & 5.6548667780 & 5.6411169080 & 5.6548667780 \\
\hline 1.0 & 6.2831853080 & 6.2470760000 & 6.2831853080 \\
\hline
\end{tabular}

Volume 10 Issue 6, June 2021 www.ijsr.net 


\section{International Journal of Science and Research (IJSR) \\ ISSN: 2319-7064 \\ SJIF (2020): 7.803}

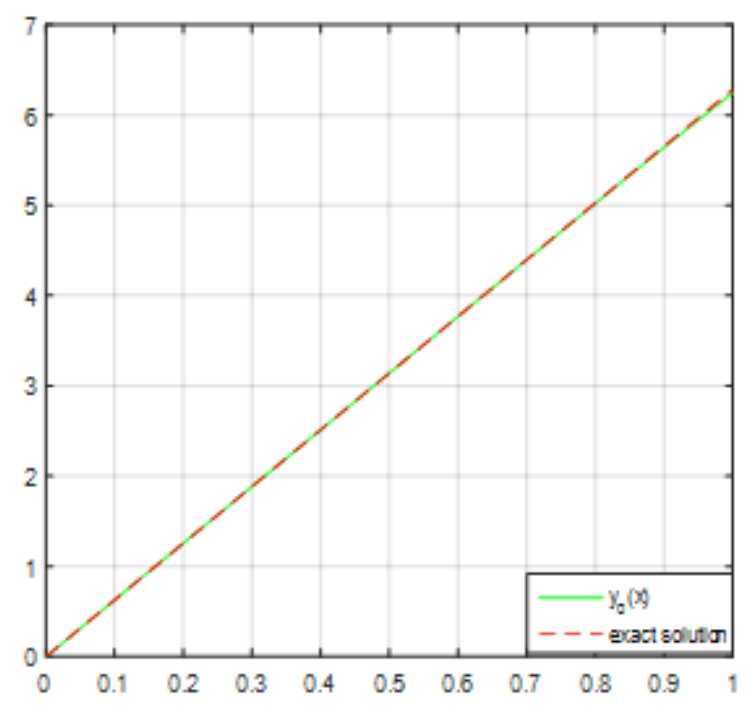

Figure 5: Comparison between exact and numerical solutions of Example 4
Example 5: Consider the following linear Fredholm integral equation of the first kind (Wazwaz 2011),

$$
y(x)=e^{x+2}-2 \int_{0}^{1} e^{x+t} y(t) d t, 0 \leq x, t \leq 1 .
$$

The exact solution of Eq. (20) is $y(x)=e^{x}$ From Eq. (20), one obtains, $f(x)=e^{x+2}$ and $K(x, t)=e^{x+t}$ which are continuous functions in the interval $[0,1]$. The numerical results shown in Table 6 , shows the effectiveness of the method as the values of $N$ increases the numerical solution becomes closer and closer to the exact solution. Figure 6 , reveals that the approximate solutions are consistent with the exact solution even for the low values of $N$. Table 7 and Figure 8 shows the calculated upper limit errors.Figure 7 shows the comparison between the exact solution and the numerical solution presented by [7].

Table 6: Comparison of the exact and numerical solutions of Example 5

\begin{tabular}{|c|c|c|c|c|c|}
\hline$x_{i}$ & Exact Solution $y\left(x_{i}\right)=e^{x_{i}}$ & $N=3, y_{3}\left(x_{i}\right)$ & $N=5, y_{5}\left(x_{i}\right)$ & $N=7, y_{7}\left(x_{i}\right)$ & $N=10, y_{10}\left(x_{i}\right)$ \\
\hline 0 & 1.000000000 & 1.012639840 & 1.000296094 & 1.000004092 & 0.9999999986 \\
\hline 0.1 & 1.105170918 & 1.119135796 & 1.105498150 & 1.105175438 & 1.105170916 \\
\hline 0.2 & 1.221402758 & 1.236770791 & 1.221764316 & 1.221407707 & 1.221402756 \\
\hline 0.3 & 1.349858808 & 1.366557464 & 1.350257434 & 1.349863952 & 1.349858803 \\
\hline 0.4 & 1.491824698 & 1.509508455 & 1.492260387 & 1.491829197 & 1.491824693 \\
\hline 0.5 & 1.648721271 & 1.666636403 & 1.649186087 & 1.648723071 & 1.648721264 \\
\hline 0.6 & 1.822118800 & 1.838953949 & 1.822587497 & 1.822113756 & 1.822118793 \\
\hline 0.7 & 2.013752707 & 2.027473733 & 2.014167623 & 2.013733346 & 2.013752696 \\
\hline 0.8 & 2.225540928 & 2.233208394 & 2.225789514 & 2.225494680 & 2.225540911 \\
\hline 0.9 & 2.459603111 & 2.457170572 & 2.459486273 & 2.459509796 & 2.459603079 \\
\hline 1.0 & 2.718281828 & 2.700372907 & 2.717471056 & 2.718110082 & 2.718281765 \\
\hline
\end{tabular}

Table 7: The comparison of residual errors (upper limit errors) corresponding to Example 5

\begin{tabular}{|l|l|}
\hline$N$ & $\bar{R}_{N}$ \\
\hline 2 & 0.3506303984 \\
\hline 3 & 0.06967382730 \\
\hline 5 & 0.00165299272 \\
\hline 7 & 0.00021365854 \\
\hline 11 & $1.0111 \mathrm{e}-07$ \\
\hline
\end{tabular}

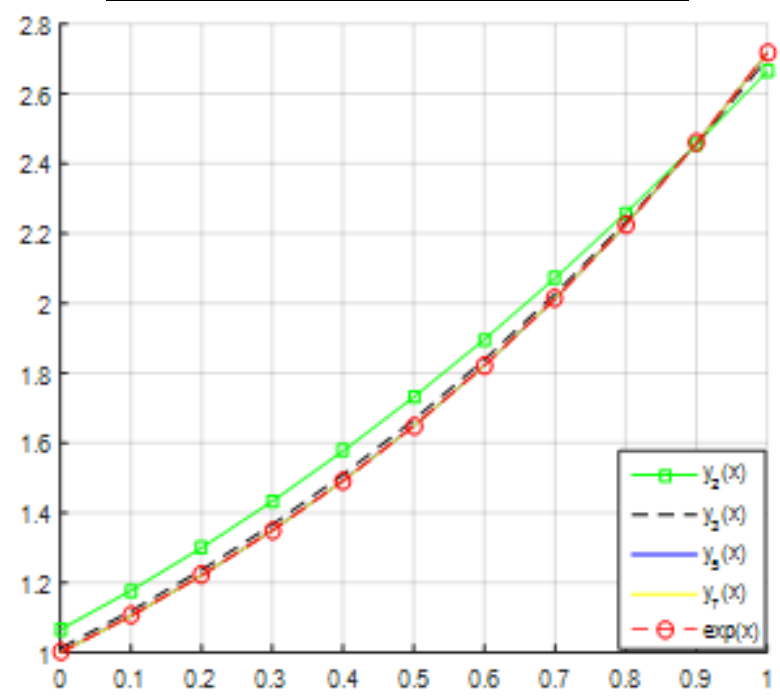

Figure 6: Comparison between exact and numerical solutions of Example 5

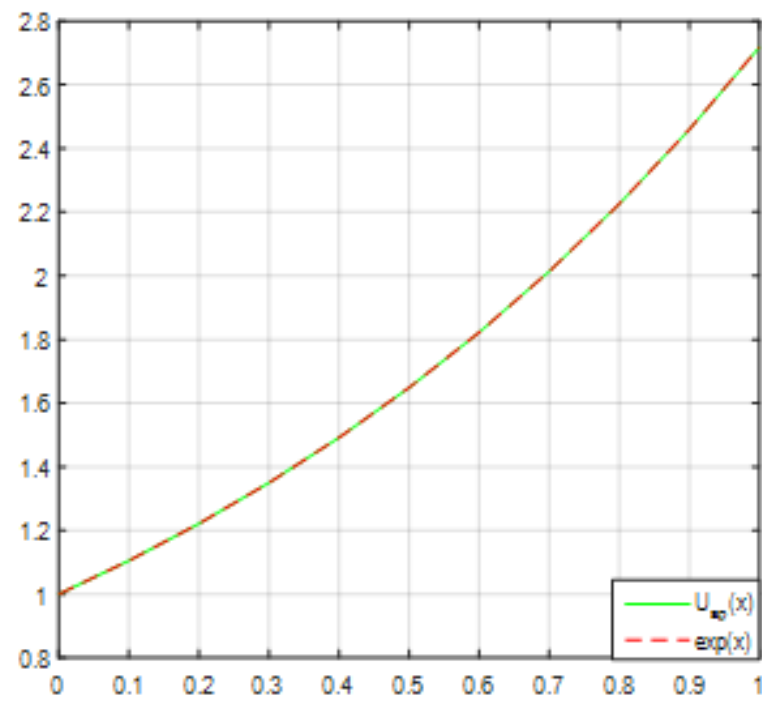

Figure 7: Comparison between exact and numerical solutionof Example 5 as presented by [7]

Volume 10 Issue 6, June 2021 www.ijsr.net

Licensed Under Creative Commons Attribution CC BY 


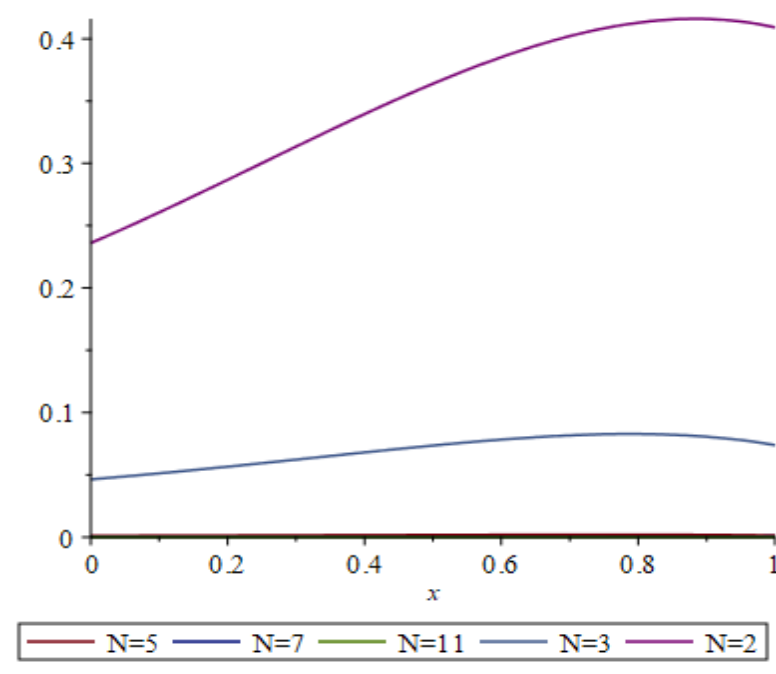

Residual error analysis figure for example 5

Figure 8: Shows the calculated upper limit errors for Example 5

\section{Conclusion}

In this study, the Pell-Lucas matrix-collocation method for solving Fredholm integral equations of the second kind is introduced. The method of residual error analysis is presented to indicate the accuracy of the solutions. The proposed method and the error analysis method are applied to some numerical examples. The comparison of the obtained numerical results with the exact solution shows that the approximate solutions become closer to the exact solution as the values $N$ increase. As evident in Example 4, this method is provides a more accurate approximation as compared to other methods. This method has alot of advantages for this type of problems, because larger matrices can be easily solved via computer programs such as MAPLE,MATLAB and MATHEMATICA. Consequently, this method can also be extended and easily applied to different types of equations.

\section{References}

[1] Donmez Demir,D., Lukonde,A.P.A., Kurkcu,O.K., Sezer, M. (2021) Pell-Lucas series approach for a class of Fredholm-type delay integro-differential equations with variable delays. Mathematical sciences, 15:55-64.

[2] Jose, M.G., Hernandez-Veron, M.A.A. (2021) A Picard-type iterative scheme for Fredholm integral equations of the second kind. Mathematics, 9:83-98.

[3] Wazwaz AM. (2011) Applications of Integral Equations. Linear and Nonlinear Integral Equations. Springer, Berlin, Heidelberg.

[4] Bulent, Y., Yasin, C. (2017) Numerical solutions of the Fredhom integral equations of the second type. New trends in Mathematical sciences, 3:284-292.

[5] Firouzdor, R., Heidarnejad, K.A., Mollaramezan, Z. (2012) Numerical solution of functional integral equations by using B-Splines. Journal of Linear and Topological Algebra, 01(01):45-53.

[6] Huaiqing, Z., Yu Chen., Xin, N. (2014) Solving the linear integral equations based on radial basis function interpolation. Journal of Applied mathematics volume 2014, article ID: 793582, 8 Pages.

[7] Ahmet, A. (2016) Numerical solution of linear and nonlinear Fredholm integral equations by using weighted mean-value theorem. Springer plus, 5:19621977.

[8] Hasib, U.M., Goutam, S. (2018) Numerical approximation of Fredholm integral equation (FIE) of $2^{\text {nd }}$ kind using Galerkin and collocation method. J. Bangladesh Math. Soc. (ISSN 1606-3694), 38:11-25.

[9] Hendi, F.A., Albugami, A.M. (2010) Numerical solution for Fredholm-Volterra integral equation of the second kind using collocation and Galerkin methods. Journal of King Saudi university (Science), 22:37-40.

[10] Silvia, A.S., Maria, I.T. (2018) Iterated PetrovGalerkin with regualar pairs for solving Fredholm integral equations of the second kind. Mathematical and computational applications, 23,73.

[11] Babasola, O.L., Irakoze, I. (2017) Collocation technique for numerical solution of integral equations with certain orthogonal basis function in interval $[0,1]$. Open access library journal, volume 4,e 4050.https://doi.org/10.4236/oalib.1104050

[12] Gümgüm S, Savaşaneril Baykuş N, Ömür Kürkçü K, Sezer M (2018) A numerical technique based on Lucas polynomials together with standard and ChebyshevLobatto collocation points for solving functional integro-differential equations involving variable delays. Sakarya University Journal of Science 22(6):1659-1668.

[13] Dönmez Demir D, Çınardalı T, Kürkçü Ömür K, Sezer M (2019) The Legendre matrix-collocation approach for some nonlinear differential equations arising in physics and mechanics. European Journal of Science and Technology 15:289-296.

[14] Şahin M, Sezer M (2018) Pell-Lucas collocation method for solving high-order functional differential equations with hybrid delays. Celal Bayar University Journal of Science 14:141-149.

[15] Baykuş Savaşaneril N, Sezer M (2017) Hybrid TaylorLucas collocation method for numerical solution of high-order,Pantograph type delay differential equations with variables delays. Appl.Math. Inf. Sci. 11(6):1795-1801.

[16] Erdem K, Yalçınbaş S, Sezer M (2013) A Bernoulli polynomial approach with residual correction for solving mixed linear Fredholm integro-differentialdifference equations. J. Differ. Equ. Appl. 19(10): 1619-1631. 